THE JOURNAL OF INFECTIOUS DISEASES - VOL. 158, NO. 4 - OCTOBER 1988

(C) 1988 by The University of Chicago. All rights reserved. $0022-1899 / 88 / 5804-0005 \$ 01.00$

\title{
Randomized Treatment of Patients with Typhoid Fever by Using Ceftriaxone or Chloramphenicol
}

\author{
Asma Islam, Thomas Butler, Samir K. Nath, \\ Nurul H. Alam, Klaus Stoeckel, Harold B. Houser, \\ and Arnold L. Smith
}

\begin{abstract}
From the International Centre for Diarrhoeal Disease Research, Bangladesh, Dhaka, Bangladesh; the Departments of Medicine and of Epidemiology and Biostatistics, Case Western Reserve University and University Hospitals of Cleveland, Cleveland, Ohio; the Biological Pharmaceutical Research Department, F. Hoffman-La Roche and Company Limited, Basel, Switzerland; and the Depariment of Pediatrics, University of Washington, Seattle, Washington
\end{abstract}

\begin{abstract}
Sixty-three patients with Salmonella typhi infections were randomly assigned to receive either ceftriaxone iv in single daily doses of $75 \mathrm{mg} / \mathrm{kg}$ for children and $3-4 \mathrm{~g}$ for adults for seven days or to receive $60 \mathrm{mg}$ of chloramphenicol $/ \mathrm{kg}$ a day orally or iv in four divided doses until defervescence and then $40 \mathrm{mg} / \mathrm{kg}$ a day to complete $14 \mathrm{~d}$. In the ceftriaxone group, one death occurred, and two of seven patients still febrile $11 \mathrm{~d}$ after starting treatment were given chloramphenicol. In the chloramphenicol group, one death and one gastrointestinal perforation occurred. The probability of remaining febrile was similar for both groups during the first seven days but was significantly greater for patients receiving ceftriaxone during the 14-d period. Patients in the chloramphenicol group were more likely to be bacteremic on day 3 . These results suggest that a seven-day course of once-daily ceftriaxone shows promise as an alternative to $14 \mathrm{~d}$ of chloramphenicol for treating typhoid fever.
\end{abstract}

Chloramphenicol has remained the drug of choice for typhoid fever for $>\mathbf{3 5}$ y because no newer antimicrobial drug has been shown to give better or more consistent clinical improvement at a comparable cost. In vitro resistance to chloramphenicol has been a rare and sporadic problem [1]. Nevertheless, there are important disadvantages $-(I)$ treatment does not prevent chronic fecal carriage of Salmonella typhi, relapses after the end of therapy, or the complications of intestinal perforation and bleeding; (2) residual mortality occurs during therapy; (3) reversible bone marrow suppression develops and rare aplastic anemia is a risk; and (4) the course of treatment requires

Received for publication 4 February 1988 and in revised form 16 May 1988.

Informed consent was obtained from the patients or from their parents or guardians, and the guidelines for human experimentation of the Ethical Review Committee of the International Centre for Diarrhoeal Disease Research, Bangladesh, were followed in the conduct of this research.

This work was supported by a grant from F. Hoffmann-La Roche and Company Limited.

We thank Drs. Keith Arnold, Michael Fernex, Bernard Surbek, and Raymond Lasserre for advice and Dr. Bernard Rowe for the Vi-phage typing.

Please address requests for reprints to Dr. Thomas Butler, Department of Internal Medicine, Texas Tech University Health Sciences Center, Lubbock, Texas 79430. administering the drug four times a day for two weeks. Shorter courses of chloramphenicol are not advised, because relapses occur in $10 \%-20 \%$ of treated cases one to two weeks after therapy.

Ceftriaxone is a newer cephalosporin antibiotic with good in vitro activity, as shown by MICs of $0.05 \mu \mathrm{g} / \mathrm{mL}$ for most tested strains of Salmonella [2]. Penetration of this antibiotic into tissues and particularly into cells $[3,4]$ indicates that it might be active against the intracellular pathogen $S$. typhi. The prolonged serum half-life of $8 \mathrm{~h}$ and biliary excretion of ceftriaxone permit less-frequent doses for enteric infections $[5,6]$. The use of ceftriaxone for treating murine typhoid showed a good therapeutic effect [7], and an open trial with 14 patients with typhoid fever in Singapore produced cures in 13 patients [8].

\section{Materials and Methods}

Selection of patients. Adults and children who reported to the International Centre for Diarrhoeal Disease Research, Bangladesh between June 1984 and December 1985 were considered for inclusion in the study. Criteria for initial inclusion were age (six months to $60 \mathrm{y}$ ), fever (for four or more days), diarrhea (at least three liquid stools in the past $24 \mathrm{~h}$ ), abdominal pain or tenderness, and $\mathrm{O}$ agglutinin titer 
$(\geqslant 80$ for $S$. typhi). Patients were excluded if they had a history of effective antimicrobial therapy within one week before hospitalization, known allergy to penicillin or cephalosporin, or other complications (including gastrointestinal hemorrhage or perforation) or jaundice. The patients were randomly assigned to receive chloramphenicol or ceftriaxone by using a table of random numbers. Only those patients with cultures of blood or stool that were positive for $S$. typhi were studied.

Assessment of patients and bacteriologic investigations. Histories and physical examinations were completed before therapy began. Rectal temperatures, pulse rates, and blood pressures were recorded at 8-h intervals. Frequency and consistency of stools were recorded every $8 \mathrm{~h}$. Stools were cultured for Salmonella, Shigella, Vibrio, Campylobacter, and enterotoxigenic Escherichia coli (ETEC). Physical examination and vital signs were followed up until the end of the 14-d stay at the hospital. On admission, blood, stool, and urine were cultured by using standard methods. Isolated strains of $S$. typhi were sent to the Enteric Reference Laboratory (Colindale, England), where Vi-phage typing was performed. The isolated strains were also tested for antimicrobial susceptibility by using agar diffusion. Ceftriaxone disks containing $30 \mu \mathrm{g}$ of antibiotic were used; a clear zone of $>16 \mathrm{~mm}$ in diameter indicated susceptibility.

Laboratory investigations. Before therapy was begun, fecal specimens were examined microscopically for leukocytes, ova, parasites, and occult blood (guaiac test). Urine was also microscopically examined. Blood was obtained for complete blood counts and for determining concentrations of creatinine and electrolytes. Serum concentrations of ceftriaxone and chloramphenicol were determined by high-performance liquid chromatography $[9,10]$ on specimens taken $24 \mathrm{~h}$ after a dose of ceftriaxone and $6 \mathrm{~h}$. after a dose of chloramphenicol on days 3 and 7 of treatment. Cultures of blood were repeated on days 3 and 14. Repeated complete blood counts and stool cultures were done on days 7 and 14 .

Therapy. After all initial diagnostic specimens had been collected, therapy was started according to one of two drug regimens. Chloramphenicol was given orally or iv in four divided doses daily for $14 \mathrm{~d}$. Adults and children received chloramphenicol (Kemicetene $^{\circledR}$; Farmitalia Carlo Erba, Italy) in doses of $60 \mathrm{mg} / \mathrm{kg}$ of body weight per day until defervescence. The dose was then reduced to $40 \mathrm{mg} / \mathrm{kg}$ per day to complete the 14-d regimen. Ceftriaxone (F. Hoff-
man-La Roche, Basel, Switzerland) was administered iv over $30 \mathrm{~min}$ in a single daily dose for seven days. Adults received 3 or $4 \mathrm{~g}$ and children received $75 \mathrm{mg} / \mathrm{kg}$ per day. We monitored adverse drug effects during therapy, such as urticaria, rashes, nausea, vomiting, and phlebitis. During the first week in the hospital, patients received no drugs other than chloramphenicol and ceftriaxone. Dexamethasone was not given.

Assessment of treatment. Patients were considered clinically cured if they survived, became afebrile without a major complication (perforation or bleeding), did not require retreatment for typhoid fever, and did not experience relapse after discharge. $\mathrm{Pa}$ tients were considered bacteriologically cured if their cultures of blood were negative for $S$. typhi at the end of treatment. The first afebrile day was defined as the first day the patient's rectal temperature dropped to $\leqslant 37.8 \mathrm{C}$ and remained there for at least $48 \mathrm{~h}$. Defervescence of patients was analyzed statistically using Kaplan-Meier estimates of probability of remaining febrile. Comparisons of estimates were done with a generalized Savage (Mantel-Cox) test (BMDP Statistical Software, Los Angeles). Diarrhea was considered to have ended when patients passed their last liquid stool. Relapse was defined as return of symptoms and isolation of $S$. typhi from the blood within two months after the start of therapy. Patients were instructed to report to the hospital if they became febrile after discharge from the hospital. Patients were also asked to return after one week from the date of discharge for follow-up examination.

\section{Results}

Characteristics of patients and bacterial strains. A total of 63 patients with blood and/or stool isolates of S. typhi were studied (29 adults and 34 children $<16$ y old). By random assignment, 31 received chloramphenicol, and 32 received ceftriaxone. The sex ratios, means of ages, and durations of fever and diarrhea before admission were similar in the two treatment groups (table 1). Frequencies of splenomegaly, hepatomegaly, coated tongue, rose spots, and delirium, obtundation (a depressed level of consciousness), or stupor were also comparable in the two groups. Among the 63 patients, $84 \%$ in the chloramphenicol group and $88 \%$ in the ceftriaxone group had cultures of blood that were positive for S. typhi. Stool cultures were positive for S. typhi in $35 \%$ of patients in the chloramphenicol group and 
Table 1. Presenting features of patients with typhoid fever, according to treatment group.

\begin{tabular}{lcc}
\hline & \multicolumn{2}{c}{ Drug treatment } \\
\cline { 2 - 3 } $\begin{array}{l}\text { Characteristic } \\
\text { of patient }\end{array}$ & Chloramphenicol & Ceftriaxone \\
\hline $\begin{array}{l}\text { No. of patients } \\
\text { (males/females) }\end{array}$ & $31(21 / 10)$ & $32(24 / 8)$ \\
Age (y) & $14.6 \pm 7.4$ & $14.7 \pm 8.2$ \\
$\begin{array}{l}\text { Duration of fever before } \\
\text { admission (d) }\end{array}$ & $10.3 \pm 4.6$ & $9.7 \pm 5.4$ \\
Duration of diarrhea before & & \\
$\quad$ admission (d) & $5.6 \pm 3.8$ & $6.7 \pm 5.9$ \\
Percentage of patients with & & \\
$\quad$ Splenomegaly & 3 & 13 \\
$\quad$ Hepatomegaly & 52 & 66 \\
$\quad$ Rose spots & 19 & 38 \\
Delirium & 26 & 28 \\
$\quad \begin{array}{l}\text { Obtundation or stupor } \\
\text { Coated tongue }\end{array}$ & 10 & 9 \\
Positive culture of blood & 65 & 69 \\
Positive stool cultures & 84 & 88 \\
\hline
\end{tabular}

* Data are numbers, means $\pm \mathrm{SD}$, and percentages. No significant differences were noted between the treatment groups $(P>.05)$.

in $56 \%$ of patients in the ceftriaxone group. In the chloramphenicol group, four patients were given the drug iv during part of the hospital course because of delirium or abdominal distention.

All $S$. typhi isolates from the 63 patients were susceptible to ampicillin, chloramphenicol, trimethoprim-sulfamethoxazole, gentamicin, kanamycin, nalidixic acid, and ceftriaxone. Strains from 45 patients were tested to determine their Vi-phage type at Colindale, England. The distribution of Vi-phage types was five of M1; four each of A and untypable Vi-strain 2; three each of 53, T, J1, and degraded Vi-strain 4; two each of degraded Vi-strain, Vi-negative strain, D1, E1, and J variant; and one each of untypable Vi-strain 1, C1, D2, F4, J5, K1, 28, 32, 46, and 51.

Other enteric pathogens identified in stools from patients in the ceftriaxone group included three with trophozoites of Entamoeba histolytica and one each of Shigella dysenteriae type 1, Shigella boydii, Vibrio cholerae, and ETEC. In the chloramphenicol group, one patient each had trophozoites of E. histolytica, $S$. boydii, and $V$. cholerae.

Outcome of therapy. Twenty-nine patients $(94 \%)$ treated with chloramphenicol and $29(91 \%)$ treated with ceftriaxone were clinically cured and discharged without gastrointestinal perforation, need for retreatment, or relapse (table 2). One death occurred in each group. In the chloramphenicol group a 20-y-old woman with a one-month history of fever and anorexia died, on the fourth hospital day, of acute bronchitis and pneumonia. In the ceftriaxone group a 25$y$-old woman with a one-week history of fever and stupor died, on the third hospital day, of pneumonia and hypotension. One patient in the chloramphenicol group developed intestinal perforation on the seventh hospital day, underwent surgical treatment, and survived. On the 11th hospital day, two patients in the ceftriaxone group who were still febrile received chloramphenicol. They both had negative cultures of blood at the time chloramphenicol was started.

The time required for defervescence ranged from 4 to $13 \mathrm{~d}$ in patients treated with chloramphenicol and from 4 to $>14 \mathrm{~d}$ in patients treated with ceftriaxone. The probability of remaining febrile was similar in the two treatment groups during the first seven days after the start of therapy, but when considering the entire $14 \mathrm{~d}$, patients treated with ceftriaxone had a higher probability of remaining febrile (figure 1; $P<.05)$. In seven patients treated with ceftriaxone who were febrile $>10 \mathrm{~d}$, two had clinical signs of pneumonia, and one had amebic trophozoites in his stool. The other patients with prolonged fever showed no signs of complications, no eosinophilia, no drug rash, and the causes of fever could not be ascertained. Cessation of diarrhea after start of treatment was more rapid for patients treated with ceftriaxone than for patients treated with chloramphenicol (mean, $2.7 \mathrm{~d}$ and $4.0 \mathrm{~d}$, respectively; $P<.05$ ).

On the eighth hospital day, one patient in the ceftriaxone group received metronidazole for amebiasis. Six weeks after the end of treatment, one pa-

Table 2. Outcomes of treatment, by treatment group.

\begin{tabular}{lcc} 
& \multicolumn{2}{c}{ Drug treatment } \\
\cline { 2 - 3 } Outcome & $\begin{array}{c}\text { Chloramphenicol } \\
(n=31)\end{array}$ & $\begin{array}{c}\text { Ceftriaxone } \\
(n=32)\end{array}$ \\
\hline Cure* & 29 & 29 \\
Death & 1 & 1 \\
Need for retreatment & 0 & 2 \\
Intestinal perforation & 1 & 0 \\
Other complications & & 3 \\
$\quad$ Pneumonia & 1 & 0 \\
\hline Peritonitis & 1 &
\end{tabular}

NOTE. Data are no. of patients.

* Patients were considered cured if they survived, became afebrile without a major complication, did not require retreatment, and did not have a relapse. 


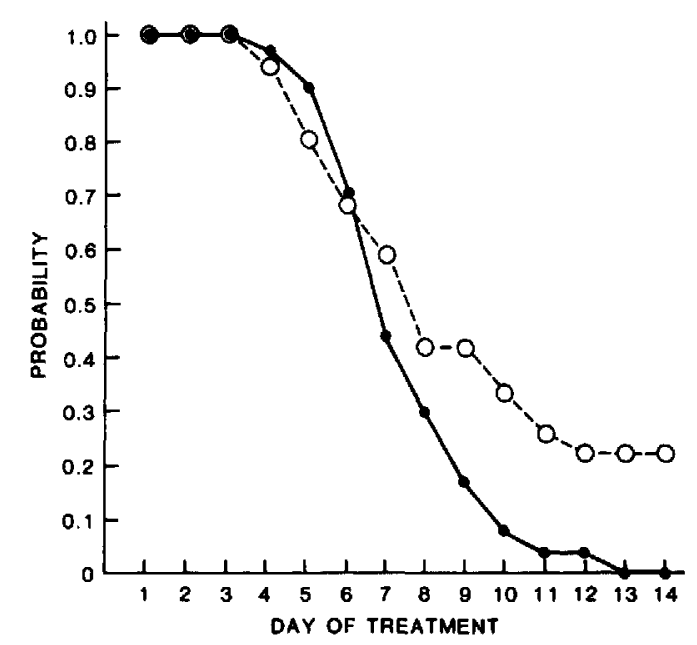

Figure 1. The probability of remaining febrile on each day after the start of treatment. Thirty-one patients were treated with chloramphenicol ( $)$ and 32 were treated with ceftriaxone (O). Two patients who died and one who developed an intestinal perforation were removed from analysis one day after the event.

tient in the ceftriaxone group experienced a reinfection with a Vi-negative strain of $S$. typhi; this strain was different from the Vi-phage type D2 that had caused the original illness.

Bacteriologic response. All of the patients treated with ceftriaxone had negative cultures of blood on the third day of treatment, whereas $52 \%$ of patients treated with chloramphenicol had positive cultures $(P<.05$ by Fisher's exact test). All cultures of blood were sterile on the 14th day after the start of therapy in both groups. Repeat stool cultures on hospital days 7 and 14 were all negative for S. typhi. Sixteen patients in the chloramphenicol group and 21 patients in the ceftriaxone group returned approximately one week after the time of discharge for examination and rectal swab cultures. All cultures were negative for S. typhi.

Drug concentrations in serum. Trough serum concentrations of ceftriaxone after daily injections on days 3 and 7 of treatment ranged from 0.9 to $56 \mu \mathrm{g} / \mathrm{mL}$. The mean concentrations on day 3 of 11.9 $\mu \mathrm{g} / \mathrm{mL}$ and on day 7 of $11.5 \mu \mathrm{g} / \mathrm{mL}$ indicated that antibiotic did not accumulate in the blood during the treatment period (figure 2). The trough serum concentrations of chloramphenicol after six-hourly treatments on days 3 and 7 ranged from 5.5 to $57 \mu \mathrm{g} / \mathrm{mL}$. Thirteen patients had trough values $>20 \mu \mathrm{g} / \mathrm{mL}$, which is the trough concentration of chlorampheni-

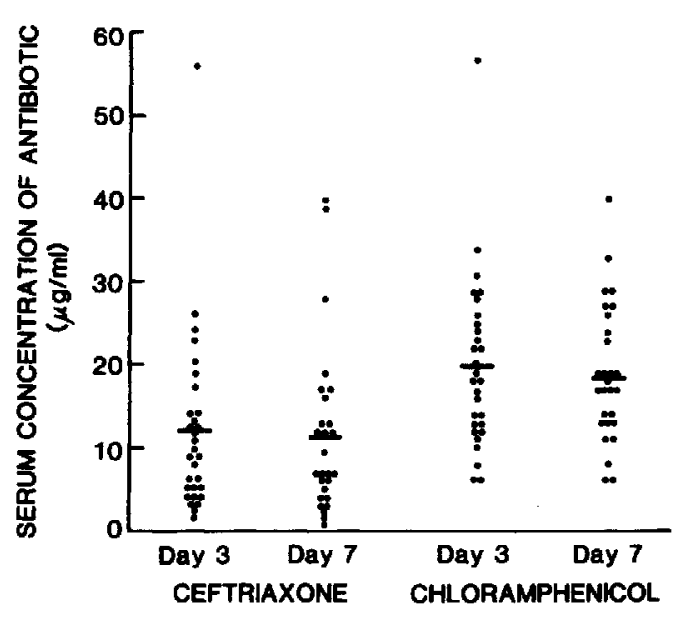

Figure 2. Trough serum concentrations of antibiotic. Ceftriaxone concentrations were measured $24 \mathrm{~h}$ after daily iv injections; chloramphenicol concentrations were measured $6 \mathrm{~h}$ after oral or iv treatments. Dots represent concentrations in individual patients. Bars show the mean concentrations on designated days.

col associated with suppression of erythropoiesis [11]. Assays of chloramphenicol in selected vials and capsules of the lots used in the study showed that the vials contained $121 \%$ of the predicted amount; the capsules stated to contain $250 \mathrm{mg}$ actually contained an average of $259.2 \mathrm{mg}$.

Hematologic repsonses. The mean hematocrit values decreased progressively during the two weeks after initiation of treatment in both treatment groups (figure 3). The mean white blood cell counts increased at day 7 and day 14 after the start of ceftriaxone treatment to levels that were significantly higher than those in the chloramphenicol group $(P<.05)$. Likewise, the mean platelet count on hospital day 14 was higher in patients treated with ceftriaxone than in patients treated with chloramphenicol $(P<.01)$.

\section{Discussion}

Our experience with treating typhoid fever with a seven-day course of ceftriaxone showed cure rates comparable to those for standard treatment with chloramphenicol. Important differences between the two treatments, however, were an earlier resolution of bacteremia and a higher proportion of patients remaining febrile for $\geqslant 10 \mathrm{~d}$ after the start of treatment in the group that received ceftriaxone. Blood levels of ceftriaxone measured $24 \mathrm{~h}$ after the previ- 

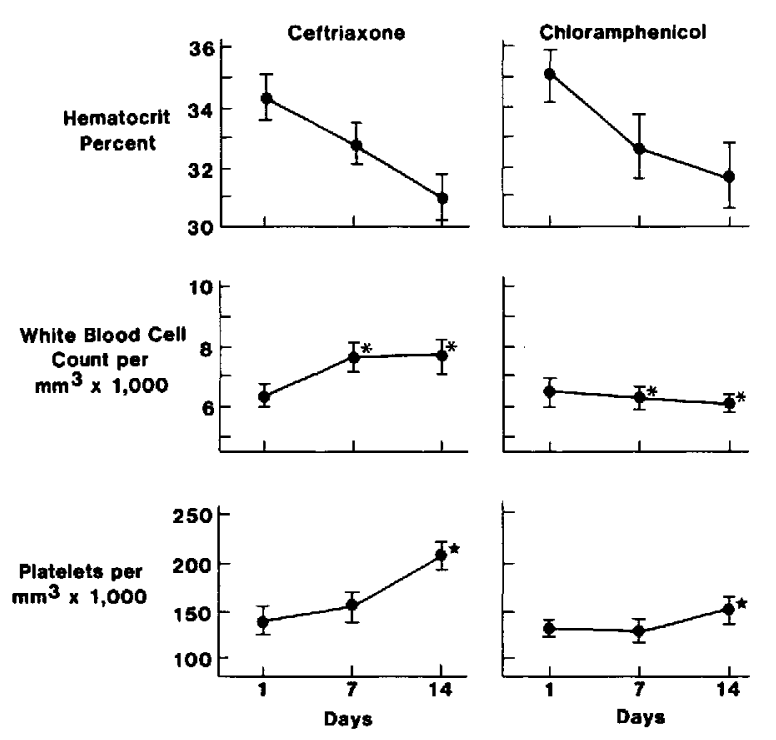

Figure 3. Hematologic responses during treatment. Values are given as means (bars, SE) before treatment and on hospital days 7 and 14. Asterisk, $P<.05 ;$ star, $P<.01$.

ous doses were in a good therapeutic range, an observation confirming that the long half-life of this drug permits one daily dose [5].

In patients treated with ceftriaxone, the time required for defervescence ranged from 4 to $>14 \mathrm{~d}$, with $23 \%$ of patients remaining febrile $>10 \mathrm{~d}$. These prolonged fevers were not explained by bacteriologic failure, inadequate blood levels of drug, or drug fever. Most of these patients were clinically improved in other respects and went on to be cured. This pattern of defervescence resembles Sanders' experience [12] with ampicillin in Indonesia. Sanders found that patients with severe typhoid fever failed to respond to ampicillin and had longer febrile courses than did patients treated with chloramphenicol; similarly, Robertson et al. [13] reported that $23 \%$ of patients in Egypt who were treated with ampicillin still had fever after $10 \mathrm{~d}$ of treatment. In Mexico, Gilman et al. [14] similarly showed that patients treated with amoxicillin defervesced more slowly than patients treated with trimethoprim-sulfamethoxazole. A possible explanation for prolonged fevers in some patients treated with ceftriaxone was that they had coinfections with other enteric bacteria or respiratory pathogens that were more effectively treated by chloramphenicol. Alternatively, ceftriaxone treatment may have reduced the burden of bacteria in the body below the level detectable by cultures of blood, but residual intracellular S. typhi, which might have been detectable by bone marrow culture [15], could have continued to cause fever while gradually being eliminated by the immune response.

The experiences of other investigators with newer cephalosporins have also indicated that these drugs may be satisfactory alternatives to chloramphenicol for treating typhoid fever. Ceftriaxone was used successfully in 13 of 14 patients with typhoid fever in an open study in Singapore [8], although three patients did not defervesce during treatment. In another open study of 19 patients with typhoid fever in Taiwan, who received ceftriaxone for only two to three days, 16 patients were judged successfully treated, whereas two patients had prolonged fevers, and one had a relapse [16]. Cefoperazone has been reported as successful treatment of typhoid fever in Haiti [17] and France [18]. Similarly, cefotaxime was given to 45 patients with typhoid fever in Korea; all defervesced in three to 14 days, and only one had a relapse [19]. Cefamandole given to nine patients in Lebanon cured six patients, with defervescence occurring after three to nine days of treatment [20].

Chloramphenicol is likely to remain the drug of choice for disease caused by chloramphenicol-susceptible strains, because chloramphenicol is cheaper, readily available for both oral and parenteral use, and regularly renders patients afebrile within $10 \mathrm{~d}$ after the start of treatment [21,22]. The role of ceftriaxone and other newer cephalosporins in typhoid fever will be in disease due to multiply resistant strains of $S$. typhi [23]. From studies of ampicillinresistant Salmonella, it can be predicted that certain cephalosporins, including ceftriaxone, cefotaxime, and cefoxitin, will have good therapeutic activity, because they are more stable in the presence of $\beta$ lactamase than are other cephalosporins [24].

The potential advantages of ceftriaxone shown in this study were a shorter course of treatment and the avoidance of bone marrow suppression by chloramphenicol, as shown by faster recovery of leukocyte and platelet counts. High serum concentrations of chloramphenicol, at which suppression of the bone marrow occurs, developed in several of our patients and could be the result of delayed hepatic metabolism of chloramphenicol in the setting of severe typhoid fever in a developing country. A portion of the high serum concentrations of chloramphenicol can be explained by the overfilling of capsules and vials by the manufacturer, an occurrence that was detected in our direct assay. Further studies are required to determine whether the advantages of ceftri- 
axone justify using this drug rather than chloramphenicol for treating patients with typhoid fever.

\section{References}

1. Edelman R, Levine MM. Summary of an international workshop on typhoid fever. Rev Infect Dis 1986;8:329-49

2. Neu HC, Meropol NJ, Fu KP. Antibacterial activity of ceftriaxone (Ro 13-9904) a B-lactamase-stable cephalosporin. Antimicrob Agents Chemother 1981;19:414-23

3. Kalager T, Digranes A, Bergan T, Solberg CO. The pharmacokinetics of ceftriaxone in serum, skin blister and thread fluid. J Antimicrob Chemother 1984;13:479-85

4. Kuhn H, Angehrn P, Havas L. Autoradiographic evidence for penetration of ${ }^{3} \mathrm{H}$-ceftriaxone (Rocephin) into cells of spleen, liver and kidney of mice. Chemotherapy 1986;32: 102-12

5. Stoeckel K, McNamara PJ, Brandt R, Plozza-Nottebrock H, Ziegler WH. Effects of concentration-dependent plasma protein binding on ceftriaxone kinetics. Clin Pharmacol Ther 1981;29:650-7

6. Arvidsson A, Alvan G, Angelin B, Borga O, Nord CE. Ceftriaxone: renal and biliary excretion and effect on the colon microflora. J Antimicrob Chemother 1982;10:207-15

7. Anton PA, Kemp JA, Butler T, Jacobs MR. Comparative efficacies of ceftriaxone, moxalactam and ampicillin in experimetal Salmonella typhimurium infection. Antimicrob Agents Chemother 1982;22:312-5

8. Ti TY, Monteiro EH, Lam S, Lee HS. Ceftriaxone therapy in bacteremic typhoid fever. Antimicrob Agents Chemother $1985 ; 28: 540-3$

9. Trautmann KH, Haefelfinger P. Determination of the cephalosporin Ro 13-9904 in plasma, urine and bile by means of ion-pair reversed phase chromatography. Journal of High Resolution Chromatography Communications 1981; 4:54-9

10. Weber AF, Opheim KE, Koup JR, Smith AL. Comparison of enzymatic and liquid chromatographic chloramphenicol assays. Antimicrob Agents Chemother 1981;19:323-5

11. McCurdy PR. Plasma concentration of chloramphenicol and bone marrow suppression. Blood 1963;21:363-72

12. Sanders WL. Treatment of typhoid fever: a comparative trial of ampicillin and chloramphenicol. Br Med J 1965;2:1226-7
13. Robertson RP, Wahab MFA, Raasch FO. Evaluation of chloramphenicol and ampicillin in Salmonella enteric fever. N Engl J Med 1968;278:171-6

14. Gilman RH, Terminel M, Levine MM, Hernandez-Mendosa $P$, Calderone E, Vasquez V, Martinez E, Snyder MJ, Hornick RB. Comparison of trimethoprim-sulfamethoxazole and amoxicillin in therapy of chloramphenicol-resistant and chloramphenicol-sensitive typhoid fever. J Infect Dis 1975;132:630-6

15. Vallenas C, Hernandez H, Kay B, Black R, Gotuzzo E. Efficacy of bone marrow, blood, stool and duodenal contents cultures for bacteriologic confirmation of typhoid fever in children. Pediatr Infect Dis 1985;4:496-8

16. Lan C-k, Cheng D-1, Lasserre $R$. Two to three days treatment of typhoid fever with ceftriaxone. Southeast Asian J Trop Med Pub HIth 1986;17:119-24

17. Pape JW, Gerdes H, Oriol L, Johnson WD Jr. Typhoid fever: successful therapy with cefoperazone. J Infect Dis 1986; 153:272-6

18. Raoult D, Gallais H, Fosse T, Xeridat B, David MF, Casanova P. Traitment de la fievre typhoide par la cefoperazone [English abstract]. Pathol Biol (Paris) 1984;32:573-5

19. Park SC, Lee CH, Kim SY, Park CH, Kim TW, Seok SE, Choi HS, Choi HR, Lee MA, Won JS. Clinical trial of cefotaxime in patients with typhoid fever. Clin Ther 1985; 7:448-51

20. Uwaydah M, Nassar NT, Harakeh H, Vartivarian S, Talhouk A, Kantarjian $H$. Treatment of typhoid fever with cefamandole. Antimicrob Agents Chemother 1984;26:426-7

21. Herzog $\mathrm{CH}$, Geddes AM. Chloramphenicol in the treatment of enteric fever. Trans R Soc Trop Med Hyg 1982;76:848-9

22. Snyder MJ, Perroni J, Gonzalez O, Woodward WE, Palomino C, Gonzalez C, Music SI, DuPont HL, Hornick RB, Woodward TE. Comparative efficacy of chloramphenicol, ampicillin, and co-trimoxazole in the treatment of typhoid fever. Lancet 1976;2:1155-7

23. Butler T, Linh NN, Arnold K, Adickman MD, Chau DM, Muoi MM. Therapy of antimicrobial resistant typhoid fever. Antimicrob Agents Chemother 1977;11:645-50

24. Cherubin CE, Eng RHK, Smith SM, Goldstein EJC. Cephalosporin therapy for Salmonellosis. Questions of efficacy and cross resistance with ampicillin. Arch Intern Med 1986; 146:2149-52 